\title{
A Top-Down LC-FTICR MS-Based Strategy for Characterizing Oxidized Calmodulin in Activated Macrophages
}

\author{
Natacha Lourette, ${ }^{\mathrm{a}, *, \ddagger}$ Heather Smallwood, ${ }^{\mathrm{a}, \mathrm{t}, \ddagger} \mathrm{Si} W \mathrm{w}^{\mathrm{b}}$

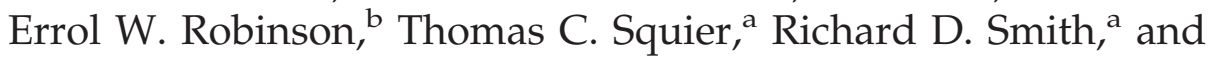 \\ Ljiljana Paša-Tolićb \\ a Fundamental and Computational Sciences Division, Pacific Northwest National Laboratory, Richland, \\ Washington, USA \\ ${ }^{\mathrm{b}}$ Environmental Molecular Sciences Laboratory, Pacific Northwest National Laboratory, Richland, \\ Washington, USA
}

\begin{abstract}
A liquid chromatography-mass spectrometry (LC-MS)-based approach for characterizing the degree of nitration and oxidation of intact calmodulin (CaM) has been used to resolve $\sim 250$ CaM oxiforms using only $500 \mathrm{ng}$ of protein. The analysis was based on high-resolution data of the intact $\mathrm{CaM}$ isoforms obtained by Fourier-transform ion cyclotron resonance mass spectrometry (FTICR MS) coupled with an on-line reversed-phase LC separation. Tentative identifications of post-translational modifications (PTMs), such as oxidation or nitration, have been assigned by matching observed protein mass to a database containing all theoretically predicted oxidation products of $\mathrm{CaM}$ and verified through a combination of tryptic peptide information (generated from bottom-up analyses) and on-line collisionally induced dissociation (CID) tandem mass spectrometry (MS/MS) at the intact protein level. The reduction in abundance and diversity of oxidatively modified CaM (i.e., nitrated tyrosines and oxidized methionines) induced by macrophage activation has been explored and semiquantified for different oxidation degrees (i.e., no oxidation, moderate, and high oxidation). This work demonstrates the power of the top-down approach to identify and quantify hundreds of combinations of PTMs for single protein target such as CaM and implicate competing repair and peptidase activities to modulate cellular metabolism in response to oxidative stress. (J Am Soc Mass Spectrom 2010, 21, 930-939) ( 2010 Published by Elsevier Inc. on behalf of American Society for Mass Spectrometry
\end{abstract}

$\mathrm{O}$ xidative stress is part of normal cellular metabolism [1]. As a central part of pathogen killing by the immune system, macrophages produce oxidizing species and use the lethal effects of oxidants to eliminate invading pathogens. However, the accelerated production of reactive oxygen species (ROS), e.g., superoxide $(\bullet \mathrm{O} 2-)$, nitric oxide $(\bullet \mathrm{NO})$, and peroxynitrite $(\mathrm{OONO}-),[2]$ and/or the impairment of mechanisms involved in maintaining normal reductive capacity within the cellular milieu can induce toxic effects that damage macromolecules and impair various cellular functions [3,4]. This imbalance between ROS pro-

Address reprint requests to Dr. L. Paša-Tolić, Environmental Molecular Sciences Laboratory, Pacific Northwest National Laboratory, 902 Battelle Blvd., P.O. Box 999 (MSIN: K8-98), Richland, WA 99352, USA. E-mail: liiljana.pasatolic@pnl.gov

* Current address: DSM Research Campus Geleen, Chemelot Gate 2/Routepunt 515, Urmonderbaan 22, 6167 RD Geleen, The Netherlands. + Current address: Department of Immunology St. Jude Children's Research Hospital, 262 Danny Thomas Place/MS 351, Memphis, TN 38105-3678, USA.

$\ddagger$ These authors contributed equally to this work. duction and cellular reductive capacity gives rise to post-translational modifications (PTMs) of intracellular DNA and proteins, which can induce mutagenic effects or changes in protein structure and/or function. Moreover, disorder in the normal redox state of the cell is now well established to be closely associated with the development of diabetes [5,6], cardiovascular diseases [7, 8], cancer [9], neurological diseases [10-13] and senescence associated with aging [14-17].

Among ROS, peroxynitrite is of particular interest because of its potential to produce diverse modifications, such as oxidation of thiols [18] or methionines [19], and nitration of tyrosines [20-22] or tryptophans $[23,24]$. Tyrosine nitration has been found extensively in various tissues in vivo, under conditions of oxidative stress, usually indicated through immunostaining with a 3-nitrotyrosine-specific antibody [25], and has been implicated in many pathophysiological conditions, e.g., kidney graft rejection [26], atherosclerosis [27], Alzheimer's disease [28], and Parkinson's disease [29]. While it is assumed that tyrosine nitration causes inhibition of
(C) 2010 Published by Elsevier Inc. on behalf of American Society for Mass Spectrometry. 1044-0305/10/\$32.00

doi:10.1016/j.jasms.2010.02.027
Published online March 22, 2010 Received November 26, 2009 Revised February 26, 2010 Accepted February 26, 2010 
protein function either by direct structural perturbation or by affecting phosphorylation, the details about the underlying nitration clearance mechanisms are lacking.

In an attempt to clarify these processes, we have examined the fate of nitrative and oxidative modifications within a model protein, calmodulin (CaM). CaM is well known to bind calcium ions, thereby transducing the calcium signal via its subsequent interactions with a multitude of target proteins, facilitating or inhibiting their activity. Among these proteins are many kinases, phosphatases, signaling proteins, and structural proteins altering a wide variety of processes including neurotransmitter release, muscle contraction, metabolism, apoptosis, inflammation, membrane protein organization, and cytoskeleton movement. Nitrotyrosine usually occurs on a fraction of the total tyrosine residues in a given protein, making it difficult to detect protein specific nitration. In addition, separation and identification of oxidized CaM species (oxiforms) generated by peroxynitrite oxidation represent a major analytical challenge. In this case, permutations in the oxidation of the nine methionines and nitration of the two tyrosines of CaM may give rise to 2048 different CaM oxiforms, with similar or identical molecular mass.

A proteomics study using Western blotting with antibodies directed against 3-nitrotyrosine identified more than 40 proteins as being potentially nitrated as a consequence of in vivo inflammatory response [30]. While Western blotting and immunoprecipitation has permitted detection of nitration on specific proteins, the procedure is often fraught with error due to lack of antibody recognition (i.e., false negative result) or nonspecific binding of antibody to many targets (i.e., false positives). Thus, the interpretation of a loss of nitrotyrosine immunoreactivity remains ambiguous, as antibodies against nitrotyrosine may be epitope-specific, varying with oxidant-induced changes in protein conformation and the overall binding affinity of the antibody.

To investigate if in vivo nitration is random or limited to specific tyrosine residues, it is necessary to develop a highly sensitive and selective method to identify specific nitration sites. Typically, nitrotyrosine quantitation has involved measuring the free nitrotyrosine either in plasma or generated following protein hydrolysis by applying various detection methods, such as LC-UV, or GCMS. These methods only provide an overall assessment of the level of free nitrated amino acid, without obtaining specific protein identification or information pertaining to the nitration site within specific proteins. Various protein separation and enrichment methods coupled with mass spectrometry (MS) based bottom-up proteomic approaches have been applied to identify nitrated proteins and locate nitration sites [31-33]. The general drawback of these approaches is the inability to discriminate between the various oxiforms (e.g., for CaM, to differentiate between iso- forms with single nitration on $\mathrm{Y}^{99}$ or $\mathrm{Y}^{138}$ and double nitration on either tyrosine). Such information is critical to determine the specificity of oxidizing agents to form PTMs and to further our understanding of the surface accessibility requirements that make specific amino acids vulnerable to modification and the related microenvironments, within proteins, that facilitate the chemistries necessary for these potentially damaging modifications to occur.

To obtain this critical information, we opted for a protein-level (top-down) approach, an emerging technology for characterizing the correlation between various PTMs within a single protein and the relative abundance of various protein forms [34-36]. Indeed, the powerful combination of high-resolution reversedphase (RP) liquid chromatography (LC) FTICR MS proved essential for the identification and quantitation of various oxidized and nitrated forms of CaM isolated from activated macrophage lysates, thereby facilitating quantitation of specific reaction products and the overall determination of modification repair on the protein.

\section{Experimental}

\section{Materials}

HPLC-grade (Optima) water, acetonitrile, isopropanol, and methanol were purchased from Fisher Scientific (Fair Lawn, NJ, USA). HPLC-grade acetic acid and trifluoroacetic acid were purchased from Aldrich (St. Louis, MO, USA). Vector pBluescript II SK was purchased from Stratagene (La Jolla, CA, USA). Antibodies used include a monoclonal antibody against glyceraldehyde-3-phosphate dehydrogenase (GAPDH) (AbCaM, Cambridge, MA, USA) and a polyclonal antibody against full-length CaM (sc-5537; Santa Cruz Biotechnology, Inc., Santa Cruz, CA, USA). The RAW 264.7 murine macrophages were obtained from Dr. Brian D. Thrall at Pacific Northwest National Laboratory.

\section{Calmodulin Mutagenesis, Expression, and Purification}

CaM [accession number MCCH (PIR database) or P02593 (SWISS-PROT database)] was cloned, expressed, and purified under standard conditions, essentially as previously described [37]. Site directed PCR-based mutagenesis was employed, as previously described [38] to add the polyhistidine tag GHHHHHHGGGGGIL on the C-terminus to allow distinction of in vitro modified CaM from endogenously produced protein.

\section{Cell Culture Procedures}

RAW 264.7 macrophages were grown to $\sim 70 \%$ confluency and cellular lysates were prepared following activation with either $10 \mathrm{ng} / \mathrm{mL}$ or $5 \mu \mathrm{g} / \mathrm{mL}$ bacterial antigen lipopolysaccharide (LPS), as previously de- 
scribed [38, 39]. Notably, all lysates were continually kept on ice, unless otherwise specified, and in the presence of a protease inhibitor cocktail (Complete Protease Inhibitor; Roche, Indianapolis, IN, USA) to prevent protein degradation.

\section{Oxidation of CaM}

Both moderately oxidized and highly oxidized CaM samples were prepared using, respectively, either a 15-fold or a 30-fold molar excess of peroxynitrite (BD Bioscience, San Diego, CA, USA) as previously described [40]. Confirmation of nitrotyrosine was determined from the isosbestic point at $381 \mathrm{~nm}$, where $\varepsilon=$ $2200 \mathrm{M}^{-1} \mathrm{~cm}^{-1}$ and may be used for quantitation at intermediate $\mathrm{pH}$ values [41].

\section{Lysate Incubation}

His-tagged CaM $(0.1 \mathrm{mg} / \mathrm{mL})$ was added to cellular lysate $(2-4 \mathrm{mg} / \mathrm{mL})$ obtained from either quiescent or activated macrophages and incubated at $37^{\circ} \mathrm{C}$ for $4 \mathrm{~h}$. Following incubation, lysate was either used for Western immunoblotting or the His-tagged CaM was affinity purified with HIS-select spin columns (Sigma-Aldrich, St. Louis, MO, USA) and dialyzed into $50 \mathrm{mM}$ $\mathrm{NH}_{4} \mathrm{HCO}_{3}(\mathrm{pH}$ 7.4) before mass spectrometry analysis.

\section{Intact Protein LC-MS}

RPLC system, similar in principle to that developed by Shen et al. [42], was used to separate the intact CaM oxiforms with $\sim 500 \mathrm{ng}$ of total protein injected on the column. LC analysis was performed under a constant pressure of 8000 psi using two ISCO (Lincoln, NE, USA) model $100 \mathrm{DM}$ high-pressure syringe pumps. The column $(80 \mathrm{~cm} \times 75 \mu \mathrm{m}$ i.d.) was packed in-house with Phenomenex (Torrance, CA, USA) Jupiter particles (C5 stationary phase; $5 \mu \mathrm{m}$ particles; $300 \AA$ pore size). The mobile phase solvents consisted of (A) $0.05 \%$ trifluoroacetic acid (TFA), $0.2 \%$ acetic acid, $5 \%$ isopropanol, $15 \%$ acetonitrile (ACN) balance water and (B) 0.1\% TFA, $9.9 \%$ water, $45 \%$ isopropanol, and $45 \%$ ACN. An exponential gradient was used for the separation, which started with $100 \%$ A and gradually increased to $70 \%$ B over $120 \mathrm{~min}$. A split was used to provide an initial flow rate through the column of $\sim 0.2 \mu \mathrm{L} / \mathrm{min}$ for $100 \%$ mobile phase A. Samples were diluted with mobile phase A, clarified by centrifugation (to protect the column from clogging) and injected through a solidphase extraction pre-column (10 $\mathrm{min})$ before RPLC-MS analysis. ESI, using a chemically etched fused-silica needle (20 $\mu \mathrm{m}$ i.d.) [43], was utilized to interface the RPLC system to a modified Bruker 12 Tesla APEX-Q FTICR mass spectrometer (Bruker Daltonics, Billerica, MA, USA) [44]. One mass spectrum was recorded every $2 \mathrm{~s}$ and the average of three spectra was used for data analysis. Here we report elution features in terms of normalized elution time (NET) by dividing the ob- served elution times by the LC run time. The resulting NET values range can from 0 to 1 .

The mass spectrometer incorporates an electrodynamic ion funnel [45], quadrupoles for collisional focusing and ion pre-selection, a hexapole for external ion accumulation, and an rf-only ion guide for ion transfer to the Bruker Infinity cell. A three-way pulsed leak valve assembly was used to introduce $\mathrm{N}_{2}$ gas during the external accumulation event (typically $0.2 \mathrm{~s}$ ). The CID (MS/MS) experiments were achieved by changing the DC offset (from 0 to $-25 \mathrm{~V}$ ) on the accumulation hexapole. The resulting fragment ions were then sent to ICR cell for the accurate mass measurements.

Intact protein RPLC-FTICR mass spectra were processed using in-house developed software (ICR-2LS available for download at http://ncrr.pnl.gov/software/), as described previously [44]. Briefly, isotopic distributions were converted to neutral masses, which were then clustered into LC-MS features, or unique mass classes (UMC, i.e., same protein detected repeatedly as it elutes from the LC column). The mass measurement accuracy for on-line LC-MS operation was estimated at $\leq 10 \mathrm{ppm}$, based on the mass variation within the UMCs. The number of distinct intact proteins was calculated by counting the UMC clusters. UMC masses were searched against a theoretical database containing all possible combinations of oxidized CaM isoforms with nitrated tyrosines and oxidized methionines for tentative intact protein identifications.

$\mathrm{CaM}$ oxiforms common between samples prior and after macrophage lysate exposure were used for alignment of individual 2D displays to provide the means to profile intact protein abundances. To align and normalize LC-MS datasets generated for comparing mixed unmodified and oxidized CaM samples, we have used a stable isotope labeled internal reference (i.e., oxidized doubly labeled $\left({ }^{13} \mathrm{C},{ }^{15} \mathrm{~N}\right) \mathrm{CaM}$, data not shown).

\section{Tryptic Digestion and RPLC-Ion Trap (IT) Peptide MS/MS Analysis}

The CaM oxidized samples were also analyzed by bottom-up proteomics. Following isolation, $\mathrm{CaM}(1.0$ $\mathrm{mg} / \mathrm{mL}$ ) was denatured (i.e., $7 \mathrm{M}$ urea, $2 \mathrm{M}$ thiourea, and $5 \mathrm{mM} \mathrm{DTT}$ ) and incubated for $30 \mathrm{~min}$ at $60^{\circ} \mathrm{C}$ before trypsin addition ( $2 \%$ by mass ratio of total protein) for overnight digestion in $0.1 \mathrm{M} \mathrm{NH}_{4} \mathrm{HCO}_{3}(\mathrm{pH}$ 8.4) and $1 \mathrm{mM} \mathrm{CaCl}_{2}$ at $37^{\circ} \mathrm{C}$. LC-MS/MS analysis was performed using a Finnigan LTQ-FT mass spectrometer (Thermo Scientific, San Jose, CA, USA). The RPLC column was manufactured in-house by slurry packing $5-\mu \mathrm{m}$ Jupiter C18 stationary phase (Phenomenex, Torrance, CA) into a $60-\mathrm{cm}$ length of $360 \mu \mathrm{m}$ o.d. $\times 150 \mu \mathrm{m}$ i.d. fused silica capillary tubing (Polymicro Technologies Inc., Phoenix, AZ, USA) incorporating a $2-\mu \mathrm{m}$ retaining screen in a $1 / 16$ in. capillary-bore union (Valco Instruments Co., Houston, TX, USA). The mobile phase consisted of $0.2 \%$ acetic acid and $0.05 \%$ TFA 


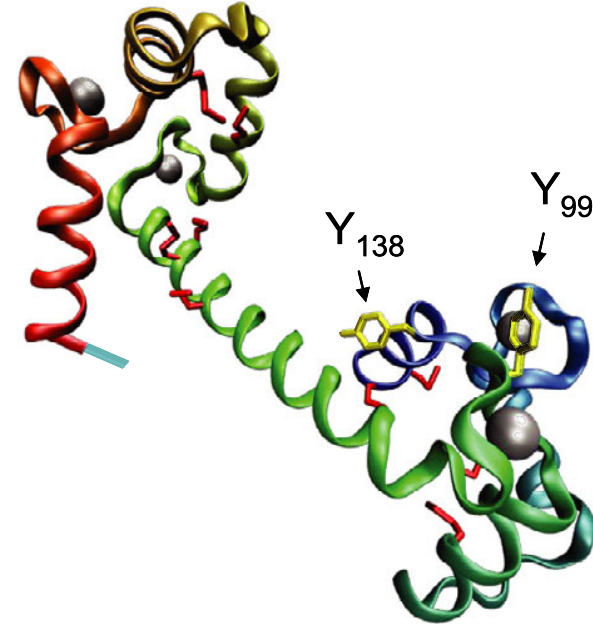

GHHHHHHGGGGGILADQLTEEQIAEFKEAFSLFDK DGDGTITTKELGTVMRSLGQNPTEAELQDMIN EVDADGNGTIDFPEFLTMMARKMKDTDSEEEI REAFRVFDKDGNG YISAAELRHVMTNLGEKLT DEEVDEMIREADIDGDGQVNYEEFVQMMTAK

Figure 1. Calmodulin 3D-structure and primary sequence. Side chains of the nine methionines and two tyrosines are respectively shown in red and orange sticks.

in water (A) and $0.1 \%$ TFA in $90 \%$ acetonitrile $/ 10 \%$ water (B).

The separation system was coupled to the mass spectrometer using an in-house manufactured interface. The heated capillary temperature and spray voltage were $200{ }^{\circ} \mathrm{C}$ and $2.2 \mathrm{kV}$, respectively. Data acquisition began $20 \mathrm{~min}$ after sample injection (15 min into the gradient) and continued for $100 \mathrm{~min}$. FTICR mass spectra (AGC $1 \times 10^{6}$ ) were collected for an $\mathrm{m} / z$ range of 400 to $2000 \mathrm{~m} / \mathrm{z}$ at a resolution of $100 \mathrm{~K}$ followed by data dependent LTQ-MS/MS. In each cycle, the five most abundant ions from MS analysis were selected for MS/MS analysis. A single repeat count and dynamic exclusion time of $60 \mathrm{~s}$ were used to discriminate against previously analyzed ions. Peptide LC-MS/MS data were processed using SEQUEST (Thermo Scientific; San Jose, CA, USA) and results were filtered as suggested by Yates et al. [46].

\section{Results and Discussion}

\section{MS Characterization of In Vitro Oxidized CaM}

The sequence and 3D structure of CaM with bound $\mathrm{Ca}^{2+}$ are shown in Figure 1. Wild type CaM is composed of 148 amino acids, including two tyrosines $\left(\mathrm{Y}_{99}\right.$, $\left.\mathrm{Y}_{138}\right)$ and nine methionines $\left(\mathrm{M}_{36}, \mathrm{M}_{51}, \mathrm{M}_{71}, \mathrm{M}_{72}, \mathrm{M}_{76}\right.$, $\left.\mathrm{M}_{109}, \mathrm{M}_{124}, \mathrm{M}_{144}, \mathrm{M}_{145}\right)$, which are surface-exposed and can therefore be nitrated (nY) and oxidized (oM), respectively, by peroxynitrite. While the addition of the polyhistadine tag added an additional 14 amino acids to the $\mathrm{N}$ terminus, we designate the first amino acid of wild type (i.e., alanine) as amino acid 1 in our Histagged $\mathrm{CaM}$, to keep with the standard designations established within the literature for this protein. Due to the two tyrosines and the overabundance of methionines in $\mathrm{CaM}$, the oxidation of $\mathrm{CaM}$ will theoretically give rise to a complex mixture containing $2048\left(2^{(9+2)}\right)$ chemically similar CaM oxiforms corresponding to only 30 different masses.

Analyzing such a complicated heterogeneous mixture of modified proteins, with similar or identical molecular masses, represents a significant challenge for any technique, including high-performance mass spectrometry. The complexity of this mixture is illustrated by the ESI-FTICR mass spectrum acquired by direct infusion of CaM treated with a 15-fold molar excess of peroxynitrite (Figure 2a). Several isotopic distributions are clearly visible in the expanded view of $16+$ charge state region (Figure $2 \mathrm{~b}$ ). The high-performance 12 Tesla FTICR mass spectrometer facilitates the differentiation of non-nitrated $\mathrm{CaM}$, singly nitrated CaM and doubly nitrated CaM, as shown Figure 2c. Even though $28 \mathrm{CaM}$ oxiforms can be identified using the direct infusion ESI-MS result displayed in Figure 2a, the exact combination of various possible $\mathrm{nY}$ and $\mathrm{oM}$ modifications (and the precise modification site) cannot be resolved. Hence, in-depth characterization of CaM oxiforms requires high-resolution separations in addition to highperformance MS.

(a)

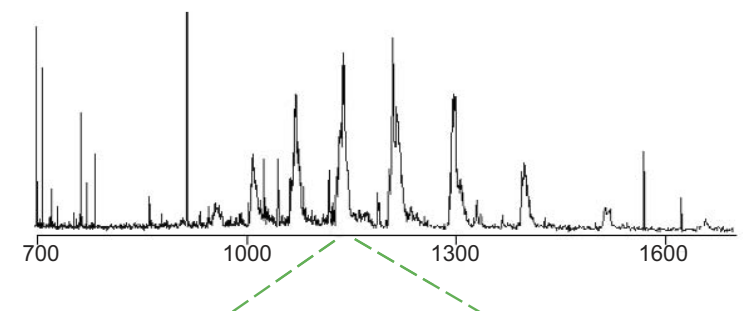

(b)

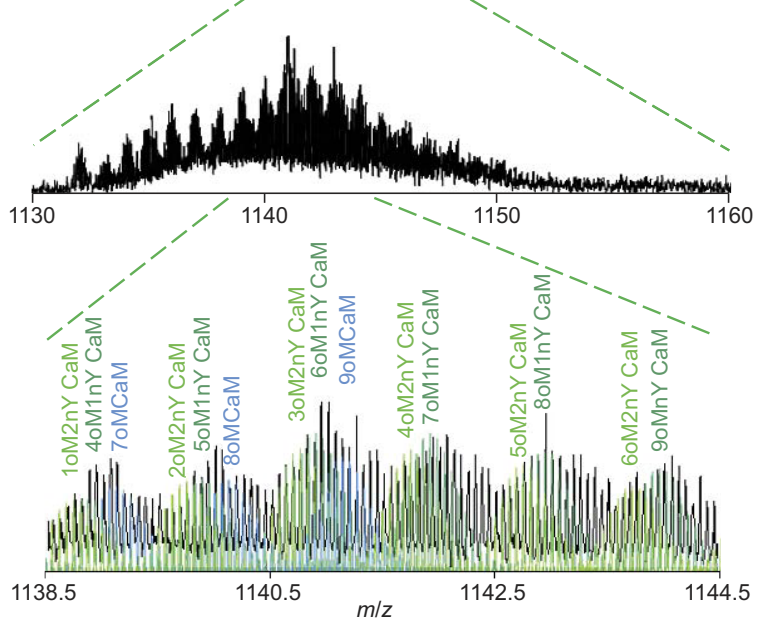

(c)

Figure 2. High-resolution mass spectrometry. ESI spectrum of CaM treated with peroxynitrite is shown in $(\mathbf{a}) ;(\mathbf{b})$ is the expanded view of the 16+ charge state of CaM isoforms and (c) is a closer zoom illustrating the isotopic resolution of different CaM oxiforms. Theoretical isotopic envelopes are overlaid as indicated. 


\section{Intact Protein LC-MS}

Our home-built nanoRPLC system enabled the separation of $\sim 250 \mathrm{CaM}$ oxiforms out of the 2048 theoretical possible isoforms using only $500 \mathrm{ng}$ of CaM treated with 15-fold molar excess of peroxynitrite injected on the column (Figure 3 and Supplemental Information). We investigated some of the elution peaks in greater detail; the spectra corresponding to each elution peak were summed to provide the mass spectra corresponding to the unique LC-MS features (Figure 3, insets). Tentative identifications were realized by matching the precise $\mathrm{m} / \mathrm{z}$ values observed for charge states $17+$ to $21+$ to the theoretical $m / z$ values calculated for CaM, including the possibility of appropriate nitrative and oxidative modifications. Recent results regarding methionine, tryptophan, and histidine oxidation support the trends in oxidation products that we observed [47].

Highly oxidized CaM elutes before the nitrated forms, thereby allowing resolution of these tightly dis- tributed masses. For example, CaM with three oxidized methionines differs only by $3 \mathrm{Da}$ from the singly nitrated CaM, but elutes about 3 min earlier in the LC separation (Figure 3). Likely, the increase in the polarity of the CaM isoforms results from an increase in the number of oxidized methionines, thereby reducing the retention of these isoforms on the column. In contrast, nitration of tyrosine results in a small increase in retention time on the RP column (compared with unmodified CaM), thus suggesting that the overall polarity of the nitrated protein is reduced.

\section{Intact Protein LC-MS/MS}

To confirm the identifications of the nitrated CaM isoforms and to characterize the sites of modification, we performed on-line multiplexed LC-MS/MS (CID) experiment (i.e., no parent ion selection). The CID

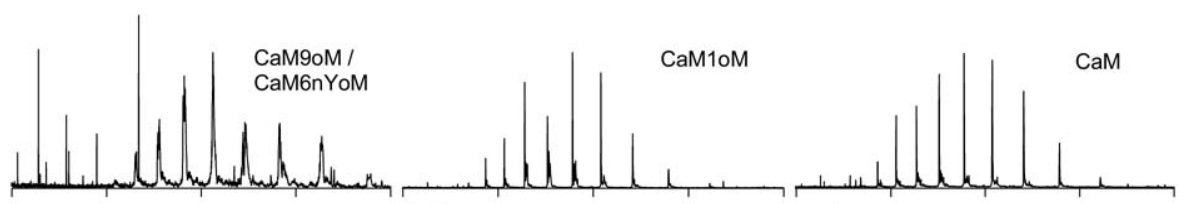

(a)

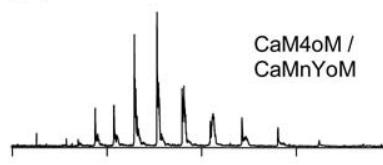

(b)

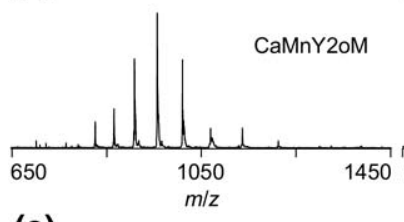

(c)

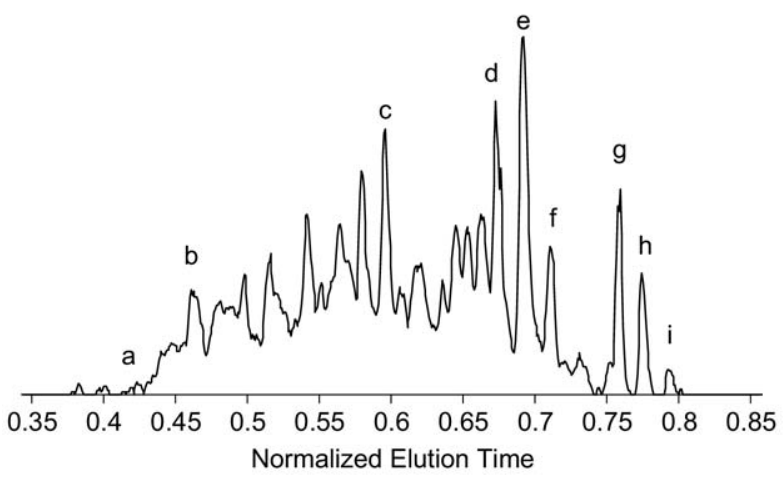

(g)

(d)

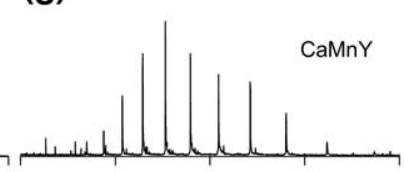

(e)

(h)

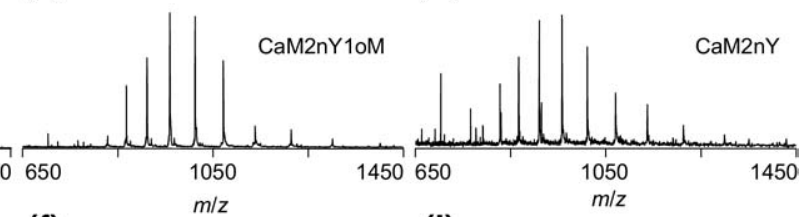

(i)

Figure 3. High-resolution separation. High-efficiency on-line microSPE-nanoLC-ESI-FTICR MS analysis of $500 \mathrm{ng}$ of CaM treated with 15 -fold molar excess of peroxynitrite. To illustrate the efficiency of the LC separation, mass spectra of different CaM oxiforms were generated from LC-MS data at the indicated elution times in the base peak chromatogram (bottom left). Peak widths artificially vary in some of the mass spectra plots due to insufficient resolution at the zoom level of the plots to observe the multiple isotope distributions resulting from varying numbers of oxiforms eluting from the RPLC column. See Figure 5 for additional plots, specifically, the LC-MS data used as the basis for the plots shown here was also used for Figure $5 \mathrm{c}$ and $\mathrm{f}$. 


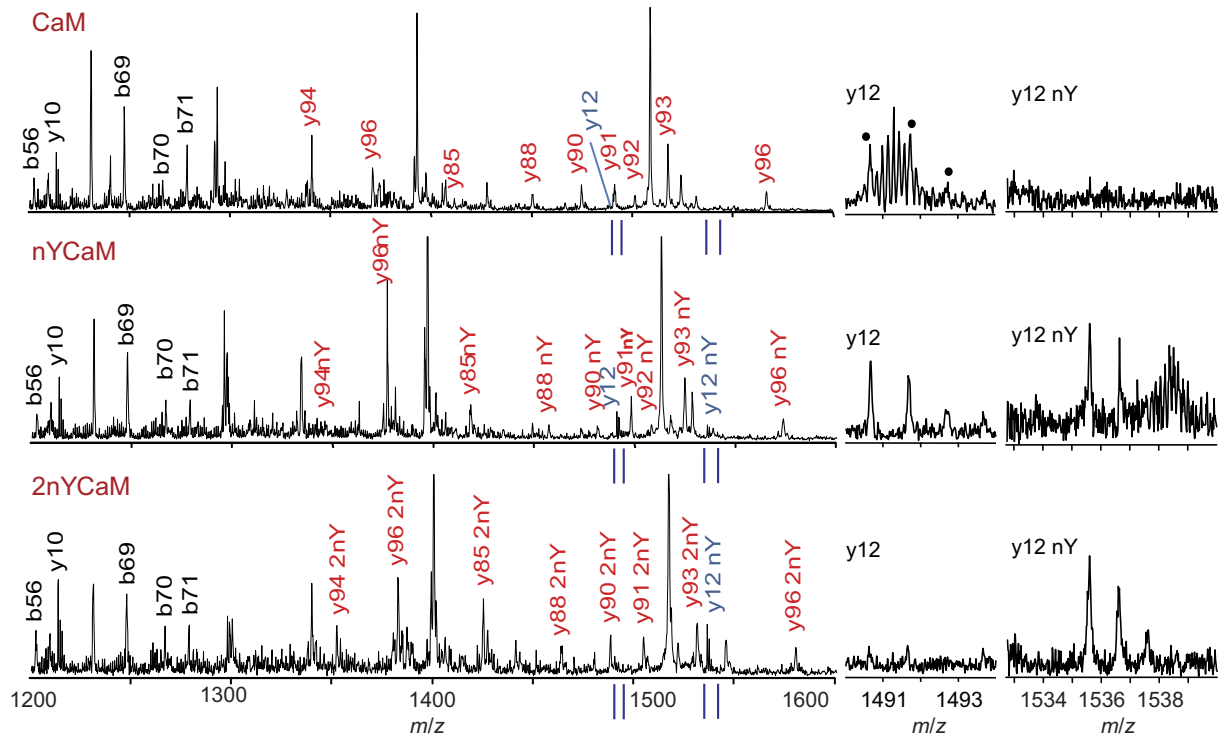

Figure 4. Identification and localization of the CaM nitration. On-line CID-MSMS spectra of CaM, nYCaM and 2nYCaM exhibit the fragments y85 to y96 increasing by 45 Da upon nitration of $Y_{99}$ and $\mathrm{Y}_{138}$. The fragment $\mathrm{y} 12$ including only $\mathrm{Y}_{138}$ in its sequence provides the nitration position.

experiment was performed with a DC offset of $-23 \mathrm{~V}$ to provide both fragment and parent ions in the same spectrum. As a result of the increase in the retention time with nitration of tyrosine, homogeneously nitrated CaM species (i.e., containing no methionine sulfoxides) can be unambiguously resolved in the LC-MS analysis,

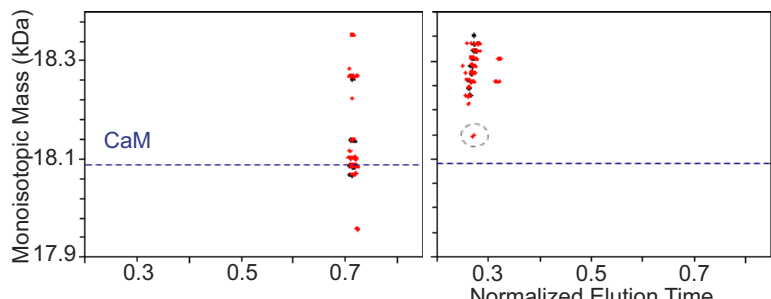

(a) (b)

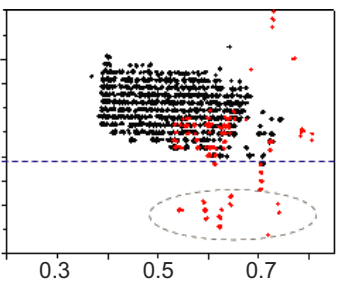

(c)

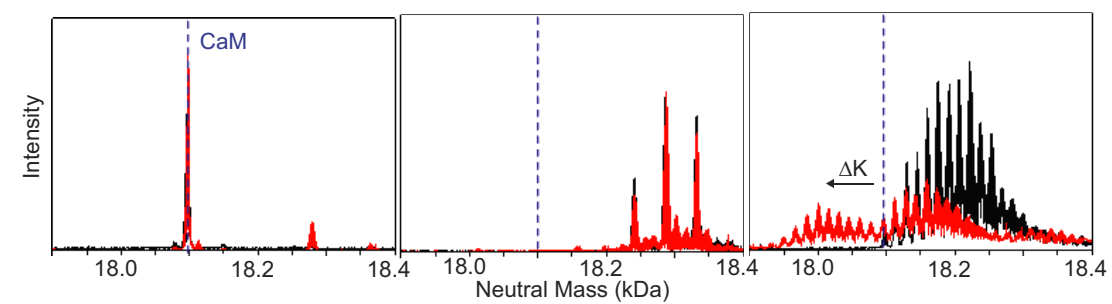

(d)

(e)

(f)

Figure 5. Repair and cleavage of modified CaM upon oxidation stress. 2D-displays of monoisotopic intact protein mass versus normalized elution time (NET) of processed MS data acquired during the RPLC separation of unoxidized His-tagged CaM (a) highly oxidized (b) or partially oxidized His-tagged CaM (c) before (black spectra) and following (red spectra) incubation with lysate from activated (i.e., exposed to LPS for 19 h) RAW 264.7 macrophages. Mass spectra were acquired with high-resolution (i.e., $\sim 150 \mathrm{~K}$ ) to ensure isotopic resolution of all detected protein species. The theoretical average mass of His-tagged CaM is 18,098.04 Da (blue dashed line), and increases by $16 \mathrm{Da}$ upon oxidation of methionines or $45 \mathrm{Da}$ upon nitration of tyrosines. Dashed grey circles indicate reference features that correspond to His-tagged CaM without $\mathrm{Lys}_{148}$ (i.e., 18,098.0 $\mathrm{Da}-128.2 \mathrm{Da}=$ $17,969.8 \mathrm{Da})$. In addition, the bottom of this figure displays the equivalent neutral mass spectra of untreated (d), highly oxidized (e), and partially oxidized (f) CaM prior (black) and post (red) macrophage lysate exposure. Moderately oxidized CaM was generated by reaction with a 15 -fold molar excess of peroxynitrite and, similarly, highly oxidized CaM was generated by reaction with a 30-fold molar excess of peroxynitrite. The LC-MS moderately oxidized CaM LC-MS data used to generate (c) and (f) was also used to generate Figure 3. 
allowing facile MS/MS data interpretation even without ion isolation. Three distinct MS/MS spectra obtained for unmodified CaM, nYCaM, and 2nYCaM are shown in Figure 4. A series of y fragments detected between y 85 and y 96 clearly indicated different levels of nitration. Tandem mass spectra obtained for the LC-MS feature assigned as a singly nitrated CaM (nYCaM) suggested that nYCaM was in fact a mixture of CaM singly nitrated at either tyrosine 99 or 138 ( $\mathrm{nY}_{99} \mathrm{CaM}$ and $\mathrm{nY}_{138} \mathrm{CaM}$ ). The presence of both unmodified and nitrated y12 fragment ions (Figure 4, middle) clearly indicates both tyrosine sites are nitrated independently.

\section{Oxidative Stress Impact on Oxidized CaM}

To investigate the fate of oxidized CaM upon exposure to the LPS-activated macrophage lysate, LC-MS analyses of three CaM preparations were performed. Plots of neutral masses versus normalized elution/retention times for no oxidation treatment, moderately oxidized (15-fold molar excess of peroxynitrite), and highly oxidized (30-fold molar excess of peroxynitrite) CaM were constructed as shown in Figure 5. Essentially, each spot in this representation corresponds to a particular CaM oxiform containing an integer number of oM and nY. Furthermore, the PTM status before (black) and after (red) interaction with macrophage proteins were compared for each preparation.

As expected, the majority of detected species from CaM with no oxidation treatment correspond to unmodified CaM $\left(\mathrm{M}_{\mathrm{r}}=18,097.45 \mathrm{Da}, \mathrm{MMA}=-1.33 \mathrm{ppm}\right)$ eluting at a normalized elution time (NET) of 0.76 , as seen in the neutral mass spectrum (Figure $5 \mathrm{~d}$ black). After exposure to the activated macrophage proteins, LC-MS analysis of the recovered CaM indicated that unmodified CaM remained the major detected component (Figure 5d red). This control experiment unequivocally demonstrated that the incubation with LPSactivated macrophage lysate did not introduce modifications to CaM and the protease inhibitor cocktail was functional throughout incubation (i.e., gross protein degradation of CaM did not occur).

Figure $5 \mathrm{c}$ and $\mathrm{f}$ show results obtained for moderately oxidized CaM, which is the most biologically relevant case. Approximately 250 CaM oxiforms (i.e., LC-MS

Table 1. Summery of identified CaM isoforms before and post macrophage exposure

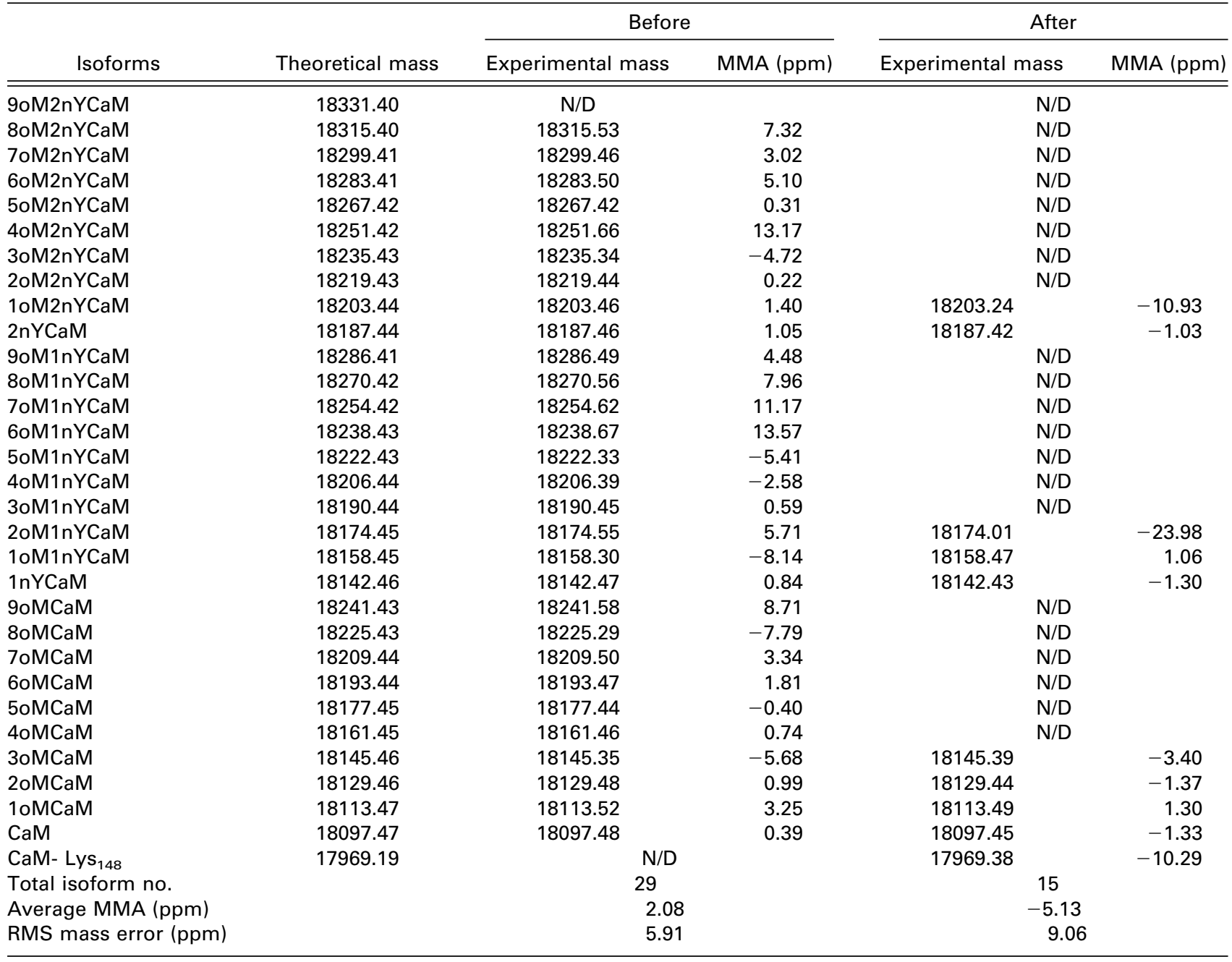


features), differing in the position and number of oM and $\mathrm{nY}$, were resolved between 0.4 and $0.8 \mathrm{NET}$ with mass ranging from 17,969 to 18,331 Da (Figure 5c black). Following incubation of moderately oxidized CaM with macrophage lysate, there is a dramatic reduction in the number of observed LC-MS features, consistent with the enzymatic reduction of both oM and nY [39]. Table 1 lists the 29 oxiforms identified for moderately oxidized CaM before exposure to the LPS-activated macrophage lysate. It is noteworthy that intact level LC-MS analysis enabled differentiation of virtually all CaM oxiforms differing in mass (29 out of 30 ) with an RMS mass measurement error less than $6 \mathrm{ppm}$. However, additional analysis, such as MS/MS at both protein and peptide level, was needed to specify the oM/nY location(s) and further differentiate between the observed 250 CaM oxiforms [39].

In addition to the substantial reduction in the overall number of observed CaM oxiforms that include $\mathrm{nY}$ (species with mass greater than 18,242 Da), a fraction of the CaM oxiforms are cleaved to generate a truncated species with a mass 128 Da below the "parent" mass. These results are consistent with prior in vivo observations that the C-terminus $\mathrm{Lys}_{148}$ is cleaved in a subpopulation of CaM isolated from oxidatively sensitive regions of the brain [48, 49]. Results from MS/MS fragmentation of the tryptic digestion of the nitrated CaM exposed to the activated macrophage lysate were also consistent with lysine C-terminal cleavage. LCMS/MS data confidently identified eight different peptides derived from the C-terminus of various CaM oxiforms containing oM or both oM and $\mathrm{nY}$ (data not shown). Two of these identified peptides were cleaved at the Lys $_{148}$, highlighting the presence of an endoge- nous peptidase activity that recognizes oxidized CaM to selectively cleave the C-terminal Lys $_{148}$ [39]. Interestingly, the bolus addition of highly oxidized protein resulted in the C-terminal lysine cleavage with little evidence of increase repair, which could be a signaling mark for degradation in instances of extreme oxidation, compared with the introduction of mildly oxidized protein which lead to the multiple repair mechanisms as well as truncation of a few species.

We also identified the following CaM oxiforms in the fully oxidized sample (Figure $5 \mathrm{~b}$ and $5 \mathrm{e}$, black): 9oMCaM $\left(\mathrm{M}_{\mathrm{r}}=18,241.58 \mathrm{Da}, 4.5 \mathrm{ppm}\right)$; 9oMnYCaM $\left(\mathrm{M}_{\mathrm{r}}=18,287.09 \mathrm{Da}, 3.9 \mathrm{ppm}\right)$, and 9oM2nYCaM $\left(\mathrm{M}_{\mathrm{r}}=\right.$ 18,332.10 Da, 4.3 ppm,). Exposure of the fully oxidized sample to the activated macrophage lysate did not have a detectable effect on the CaM oxiforms, with the exception of the appearance of a new species at $\mathrm{M}_{\mathrm{r}}=$ $18,159.07 \mathrm{Da}$ and a NET $=0.42$. These species were also tentatively identified as 9oMnYCaM (MMA $=11.9$ ppm) with C-terminal Lys $_{148}$ cleavage. Bottom-up LCMS/MS results of fully oxidized CaM sample were also consistent with the top-down results (data not shown).

\section{Semiquantitative Analysis of the Nitration Clearance}

In an attempt to quantify the changes in the oxiforms following exposure of moderately oxidized CaM to the activated macrophage lysate, the abundances of all detected species were normalized to the abundance of unmodified CaM. The 2D display of mass versus retention time was arbitrarily divided into five distinctive regions (Figure 6) covering homogeneously oxidized

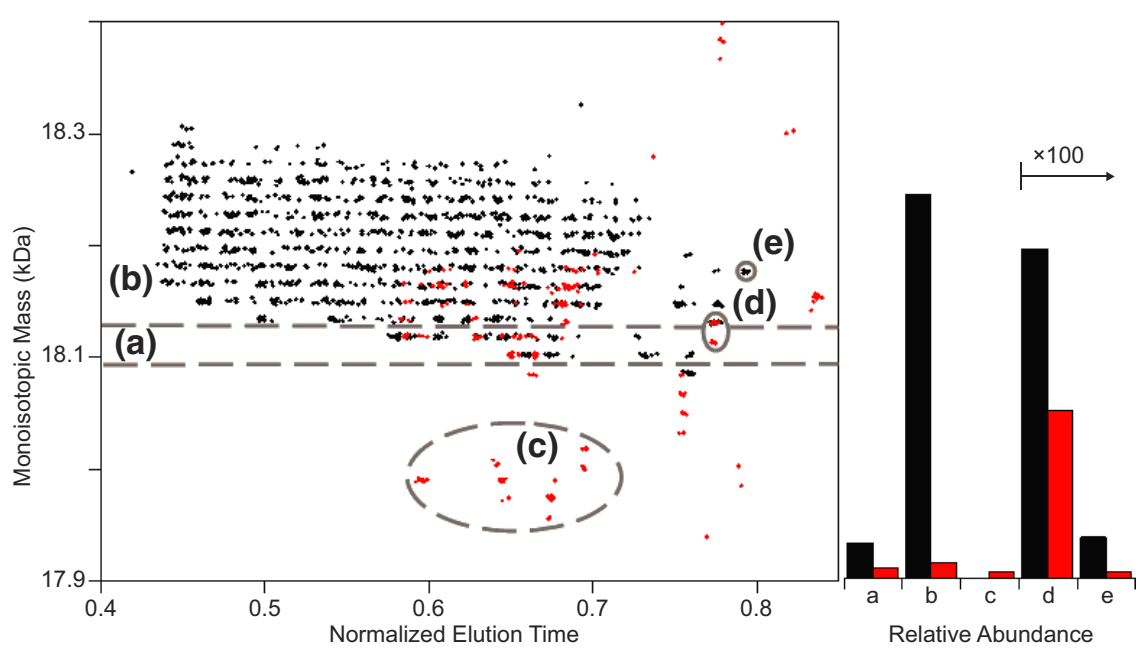

Figure 6. Semiquantification of the denitrase activity. 2D-display (left) of monoisotopic intact protein mass versus normalized elution time (NET) highlights resolved intact protein masses of individual oxidized CaM species that are indicators of denitrase activity. CaM species detected before (black) and following (red) incubation with activated macrophage lysate from (a) CaM and oxidized CaM; (b) nitrated and oxidized CaM; (c) CaM-Lys 148 and oxidized CaM-Lys ${ }_{148} ;$ (d) nYCaM, and (e) 2nYCaM. The panel at the right displays the relative abundance of CaM within each of these five areas before (black) and after (red) exposure to activated macrophage lysate. 
CaM (oMCaM), heterogeneous mixture of oxidized and nitrated CaM (oMnYCaM), truncated oxiforms; homogeneously nitrated CaMs (nYCaM), and 2nYCaM. The changes in the abundance of CaM oxiforms in each of these regions following incubation of oxidized CaM with proteins from activated macrophages are depicted in Figure 6. The most dramatic change was for the oxidized CaM group, which was reduced by $70 \%$ of its original intensity. In parallel, the combined nitrated and oxidized CaM decreases by $95 \%$ and was further modified by the loss of the C-terminal Lys, resulting in several oxiforms-Lys ${ }_{148}$. Also of note was a $50 \%$ and $80 \%$ reduction of nYCaM and $2 \mathrm{nYCaM}$, respectively. These results have profound implications. They verify the previous finding of a large reduction of CaM secondary structure following oxidative stress that is related to the oxidation of methionines near the $\mathrm{C}$ terminus $\left(\mathrm{M}_{144}\right.$ or $\left.\mathrm{M}_{145}\right)$, nitration of tyrosines, or proteolysis of Lys $_{148}$, which are all likely to disrupt the structure of $\mathrm{CaM}$ and diminish its calcium affinity and/or and target protein binding. These results also demonstrate a role methionine sulfoxide reductase and putative denitrase activity [39] in activated macrophages that functions to restore $\mathrm{CaM}$ to its native structure necessary for efficient calcium signaling. Further, the lack of repair of the heavily oxidized protein, in favor of the C-terminal lysine cleavage, is likely to represent a signaling queue for degradation of CaM following a bolus of ROS resulting in terminal oxidation of the protein.

\section{Conclusion}

Reversed-phase liquid chromatography (RPLC) separations coupled with a 12 Tesla FTICR mass spectrometry were applied to profile the changes in oxidized and nitrated CaM species related to conditions of oxidative stress (i.e., following macrophage activation by LPS). Hundreds of the CaM oxiforms induced by peroxynitrite have been detected. Protein abundance profiling demonstrated a dramatic reduction of the nitrated and oxidized forms post macrophage exposure. In addition, a modification dependent C-terminal lysine cleavage was observed and suggested as a reliable biomarker for oxidative stress. Further, the prevalence of cleavage activity in the highly oxidized sample, over repair, suggests this as a new degradative signal found on highly oxidized CaM. The high resolving power and mass measurement accuracy of our intact protein platform proved to be essential for comprehensive profiling and quantitative assessment of the multitude of PTMs in the oxidized and nitrated CaM. These data suggest competing pathways involving repair enzymes and proteases, which together modulate calcium signaling in macrophages through the modulation of CaM function.

\section{Acknowledgments}

Portions of this work were supported by the National Center for Research Resources (RR 018522) and the U.S. Department of Energy (DOE) Office of Biological and Environmental Research. Work was performed in the Environmental Molecular Science Laboratory, a DOE national scientific user facility located on the campus of Pacific Northwest National Laboratory (PNNL) in Richland, Washington. PNNL is a multi-program national laboratory operated by Battelle for the DOE under contract DE-AC0576RLO 1830.

\section{Appendix A \\ Supplementary Material}

Supplementary material associated with this article may be found in the online version at doi:10.1016/ j.jasms.2010.02.027.

\section{References}

1. Cecarini, V.; Gee, J.; Fioretti, E.; Amici, M.; Angeletti, M.; Eleuteri, A. M.; Keller, J. N. Protein Oxidation and Cellular Homeostasis: Emphasis on Metabolism. Biochim. Biophys. Acta 2007, 1773(2), 93-104.

2. Ullrich, V.; Kissner, R. Redox Signaling: Bioinorganic Chemistry at Its Best. J. Inorg. Biochem. 2006, 100(12), 2079-2086.

3. Mecocci, P.; Fano, G.; Fulle, S.; Mac Garvey, U.; Shinobu, L.; Polidori, M. C.; Cherubini, A.; Vecchiet, J.; Senin, U.; Beal, M. F. Age-Dependent Increases in Oxidative Damage to DNA, Lipids, and Proteins in Human Skeletal Muscle. Free Radical Biol. Med. 1999, 26(3/4), 303-308.

4. England, T.; Beatty, E.; Rehman, A.; Nourooz-Zadeh, J.; Pereira, P.; O'Reilly, J.; Wiseman, H.; Geissler, C.; Halliwell, B. The Steady-State Levels of Oxidative DNA Damage and of Lipid Peroxidation (F2Isoprostanes) Are Not Correlated in Healthy Human Subjects. Free Radical Res. 2000, 32(4), 355-362.

5. Ahmed, N.; Babaei-Jadidi, R.; Howell, S. K.; Beisswenger, P. J.; Thornalley, P. J. Degradation Products of Proteins Damaged by Glycation, Oxidation, and Nitration in Clinical Type 1 Diabetes. Diabetologia 2005, 48(8), 1590-1603.

6. Pacher, P.; Szabo, C. Role of Peroxynitrite in the Pathogenesis of Cardiovascular Complications of Diabetes. Curr. Opin. Pharmacol. 2006, 6(2), 136-141.

7. Kanski, J.; Behring, A.; Pelling, J.; Schoneich, C. Proteomic Identification of 3-Nitrotyrosine-Containing Rat Cardiac Proteins: Effects of Biological Aging. Am. J. Physiol. Heart Circ. Physiol. 2005, 288(1), H371-H381.

8. Turko, I. V.; Murad, F. Protein Nitration in Cardiovascular Diseases. Pharmacol. Rev. 2002, 54(4), 619-634.

9. Halliwell, B. Oxidative Stress and Cancer: Have We Moved Forward? Biochem. J. 2007, 401(1), 1-11.

10. Giasson, B. I.; Ischiropoulos, H.; Lee, V. M.; Trojanowski, J. Q. The Relationship Between Oxidative/Nitrative Stress and Pathological Inclusions in Alzheimer's and Parkinson's Diseases. Free Radical Biol. Med. 2002, 32(12), 1264-1275.

11. Halliwell, B. Oxidative Stress and Neurodegeneration: Where Are We Now? J Neurochem. 2006, 97(6), 1634-1658.

12. Ischiropoulos, H.; Beckman, J. S. Oxidative Stress and Nitration in Neurodegeneration: Cause, Effect, or Association? J Clin. Invest. 2003, 111(2), 163-169.

13. Sacksteder, C. A.; Qian, W. J.; Knyushko, T. V.; Wang, H.; Chin, M. H.; Lacan, G.; Melega, W. P.; Camp, D. G. II; Smith, R. D.; Smith, D. J.; Squier, T. C.; Bigelow, D. J. Endogenously Nitrated Proteins in Mouse Brain: Links to Neurodegenerative Disease. Biochemistry 2006, 45(26), 8009-8022.

14. Beal, M. F. Oxidatively Modified Proteins in Aging and Disease. Free Radical Biol. Med. 2002, 32(9), 797-803.

15. Finkel, T.; Holbrook, N. J. Oxidants, Oxidative Stress, and the Biology of Aging. Nature 2000, 408(6809), 239-247.

16. Harman, D. The Free Radical Theory of Aging. Antioxid. Redox Signal 2003, 5(5), 557-561.

17. Stadtman, E. R. Protein Oxidation and Aging. Science 1992, 257(5074), $1220-1224$

18. Quijano, C.; Alvarez, B.; Gatti, R. M.; Augusto, O.; Radi, R. Pathways of Peroxynitrite Oxidation of Thiol Groups. Biochem. J. 1997, 322(Pt 1), 167-173.

19. Pryor, W. A.; Jin, X.; Squadrito, G. L. One- and Two-Electron Oxidations of Methionine by Peroxynitrite. Proc. Natl. Acad. Sci. U.S.A. 1994, 91(23), 11173-11177.

20. Daiber, A.; Herold, S.; Schoneich, C.; Namgaladze, D.; Peterson, J. A.; Ullrich, V. Nitration and Inactivation of Cytochrome P450BM-3 by Peroxynitrite Stopped-Flow Measurements Prove Ferryl Intermediates. Eur. J. Biochem. 2000, 267(23), 6729-6739. 
21. Radi, R. Nitric Oxide, Oxidants, and Protein Tyrosine Nitration. Proc. Natl. Acad. Sci. U.S.A. 2004, 101(12), 4003-4008.

22. Ramezanian, M. S.; Padmaja, S.; Koppenol, W. H. Nitration and Hydroxylation of Phenolic Compounds by Peroxynitrite. Chem. Res. Toxicol. 1996, 9(1), 232-240.

23. Kato, Y.; Kawakishi, S.; Aoki, T.; Itakura, K.; Osawa, T. Oxidative Modification of Tryptophan Residues Exposed to Peroxynitrite. Biochem. Biophys. Res. Commun. 1997, 234(1), 82-84

24. Maskos, Z.; Rush, J. D.; Koppenol, W. H. The Hydroxylation of Tryptophan. Arch. Biochem. Biophys. 1992, 296(2), 514-520.

25. Aulak, K. S.; Miyagi, M.; Yan, L.; West, K. A.; Massillon, D.; Crabb, J. W.; Stuehr, D. J. Proteomic Method Identifies Proteins Nitrated In Vivo During Inflammatory Challenge. Proc. Natl. Acad. Sci. U.S.A. 2001, 98(21), 12056-12061.

26. MacMillan-Crow, L. A.; Crow, J. P.; Thompson, J. A. PeroxynitriteMediated Inactivation of Manganese Superoxide Dismutase Involves Nitration and Oxidation of Critical Tyrosine Residues. Biochemistry 1998, 37(6), 1613-1622.

27. Zou, M. H.; Leist, M.; Ullrich, V. Selective Nitration of Prostacyclin Synthase and Defective Vasorelaxation in Atherosclerotic Bovine Coronary Arteries. Am. J. Pathol. 1999, 154(5), 1359-1365.

28. Smith, M. A.; Richey Harris, P. L.; Sayre, L. M.; Beckman, J. S.; Perry, G. Widespread Peroxynitrite-Mediated Damage in Alzheimer's Disease. J. Neurosci. 1997, 17(8), 2653-2657.

29. Pennathur, S.; Jackson-Lewis, V.; Przedborski, S.; Heinecke, J. W. Mass Spectrometric Quantification of 3-Nitrotyrosine, Orthotyrosine, and $0, o^{\prime}$-Dityrosine in Brain Tissue of 1-Methyl-4-Phenyl-1,2,3, 6Tetrahydropyridine-Treated Mice, a Model of Oxidative Stress in Parkinson's Disease. J Biol. Chem. 1999, 274(49), 34621-34628.

30. Aulak, K. S.; Miyagi, M.; Yan, L.; West, K. A.; Massillon, D.; Crabb, J. W.; Stuehe, D. J. Proteomic Method Identifies Proteins Nitrated In Vivo During Inflammatory Challenge. Proc. Natl. Acad. Sci. U.S.A. 2001, 98(21), 12056-12061.

31. Turko, I. V.; Murad, F. Mapping Sites of Tyrosine Nitration by MatrixAssisted Laser Desorption/Ionization Mass Spectrometry. Methods Enzymol. 2005, 396, 266-275.

32. Zhang, Q.; Qian, W.; Knyushko, T. V.; Clauss, T. R.; Purvine, S. O.; Moore, R. J.; Sacksteder, C. A.; Chin, M. H.; Smith, D. J.; Camp, D. G. II; Bigelow, D. J.; Smith, R. D. A Method for Selective Enrichment and Analysis of Nitrotyrosine-Containing Peptides in Complex Proteome Samples. J. Proteome Res. 2007, 6(6), 2257-2268.

33. Lee, J. R.; Lee, S. J.; Kim, T. W.; Kim, J. K.; Park, H.S.; Kim, D. E.; Kim K. P.; Yeo, W. S. Chemical Approach for Specific Enrichment and Mass Analysis of Nitrated Peptides. Anal, Chem. 2009, 81(16), 6620-6626.

34. McLafferty, F. W.; Fridriksson, E. K.; Horn, D. M.; Lewis, M. A.; Zubarev, R. A. Techview: Biochemistry. Biomolecule Mass Spectrometry. Science 1999, 284(5418), 1289-1290.

35. Siuti, N.; Kelleher, N. Decoding Protein Modifications Using Top-Down Mass Spectrometry. Nat. Methods 2007, 4(10), 817-821.
36. Breuker, K.; Jin, M.; Han, X.; Jiang, H.; McLafferty, F. Top-Down Identification and Characterization of Biomolecules by Mass Spectrometry. J. Am. Soc. Mass Spectrom. 2008, 19(8), 1045-1053.

37. Strasburg, G. M.; Hogan, M.; Birmachu, W.; Thomas, D. D.; Louis, C. F. Site-Specific Derivatives of Wheat Germ Calmodulin. Interactions with Troponin and Sarcoplasmic Reticulum. J. Biol. Chem. 1988, 263(1), 542-548.

38. Smallwood, H. S.; Galeva, N. A.; Bartlett, R. K.; Urbauer, R. J.; Williams, T. D.; Urbauer, J. L.; Squier, T. C. Selective Nitration of Tyr99 in Calmodulin as a Marker of Cellular Conditions of Oxidative Stress. Chem. Res. Toxicol. 2003, 16(1), 95-102.

39. Smallwood, H. S.; Lourette, N. M.; Boschek, C. B.; Bigelow, D. J.: Smith, R. D.; Paša-Tolić, L.; Squier, T. C. Identification of a Denitrase Activity Against Calmodulin in Activated Macrophages Using High-Field LCFTICR Mass Spectrometry. Biochemistry 2007, 46(37), 10498-10505.

40. Richman, P. G.; Klee, C. B. Conformation-Dependent Nitration of the Protein Activator of Cyclic Adenosine 3',5'-Monophosphate Phosphodiesterase. Biochemistry 1978, 17(5), 928-935.

41. Riordan, J. F.; Sokolovsky, M.; Vallee, B. L. The Functional Tyrosyl Residues of Carboxypeptidase A. Nitration with Tetranitromethane. Biochemistry 1967, 6(11), 3609-3617.

42. Shen, Y. Zhao, R.; Belov, M. E.; Conrads, T. P.; Anderson, G. A.; Tang K.; Paša-Tolić, L.; Veenstra, T. D.; Lipton, M. S.; Udseth, H. R.; Smith, R. D. Packed Capillary Reversed-Phase Liquid Chromatography with High-Performance Electrospray Ionization Fourier Transform Ion Cyclotron Resonance Mass Spectrometry for Proteomics. Anal. Chem. 2001, 73(8), 1766-1775

43. Kelly, R. T.; Page, J. S.; Luo, Q.; Moore, R. J.; Orton, D. J.; Tang, K.; Smith, R. D. Chemically Etched Open Tubular and Monolithic Emitters for Nanoelectrospray Ionization Mass Spectrometry. Anal. Chem. 2006, 78(22), 7796-7801.

44. Sharma, S.; Simpson, D. C.; Tolić, N.; Jaitly, N.; Mayampurath, A. M.; Smith, R. D.; Paša-Tolić, L. Proteomic Profiling of Intact Proteins Using WAX-RPLC 2-D Separations and FTICR Mass Spectrometry. J. Proteome Res. 2007, 6(2), 602-610.

45. Shaffer, S. A.; Prior, D. C.; Anderson, G. A.; Udseth, H. R.; Smith, R. D. An Ion Funnel Interface for Improved Ion Focusing and Sensitivity Using Electrospray Ionization Mass Spectrometry. Anal. Chem. 1998, 70(19), 4111-4119.

46. Washburn, M. P.; Wolters, D.; Yates, J. R. III. Large-Scale Analysis of the Yeast Proteome by Multidimensional Protein Identification Technology. Nat. Biotechnol. 2001, 19(3), 242-247.

47. Ji, J. A.; Zhang, B. Y.; Cheng, W.; Wang, Y. J. Methionine, Tryptophan, and Histidine Oxidation in a Model Protein, PTH: Mechanisms and Stabilization. J. Pharmaceut. Sci. 2009, 98(12), 4485-4500.

48. Isobe, T.; Ishioka, N.; Okuyama, T. Isolation and Characterization of des(Ala-Lys)Calmodulin in Porcine Brain. Biochem. Biophys. Res. Commun. 1981, 102(1), 279-286.

49. Murtaugh, T. J.; Wright, L. S.; Siegel, F. L. Post-Translational Modification of Calmodulin in Rat Brain and Pituitary. J. Neurochem. 1986, 47(1), 164-172. 